\title{
Estimation of self-fertilization rate and allelic frequencies in diploidized tetraploids
}

\author{
PHILIP W. HEDRICK, ELMARIE S. HUTCHINSON* \& MICHAEL MESLER $\dagger$ \\ Department of Biology, Pennsylvania State University, University Park, PA 16802, *Bodega Marine Laboratory, Bodega \\ Bay, CA 94923 and $\div$ Department of Biology, Humboldt State University, Arcata, CA 95521, USA
}

\begin{abstract}
A methodology for the estimation of the self-fertilization rate and allelic frequencies in diploidized tetraploids is developed. Data from both a population survey and independent progeny arrays from homozygous parents are generally necessary for maximum likelihood estimates. If progeny arrays from a random population sample are examined, then data from the resulting six phenotypic categories may be used. An example using the first approach is given using data from the Menzies' wallflower, an endangered species in California.
\end{abstract}

Keywords: Erysimum menziesii, polyploidy, self-fertilization rate.

\section{Introduction}

The estimation of basic population-genetic parameters, such as allelic frequencies, in polyploids is often complicated (e.g. Waples, 1988). Moreover, another level of complexity is added when a certain amount of self-fertilization cannot be ruled out because Hardy-Weinberg proportions can then no longer be assumed in the estimation procedures. Because both polyploidy and self-fertilization are common in plants, self-fertilizing polyploid species may not be unusual. In a partially self-fertilizing polyploid, duplicated loci (isoloci, see Discussion) may share the same codominant allelic variants, such as those assayed for by starch-gel electrophoresis. We give below procedures that can be used to estimate jointly allelic frequencies and rates of self-fertilization in this case and illustrate these techniques using data from Erysimum menziesil, the Menzies' wallflower, which is listed as an endangered species in California.

\section{Model}

It is assumed in the following discussion that a diploidized tetraploid is being examined, i.e. the loci in the different genomes that formed the tetraploid assort independently. With the same two allelic variants in each genome, $\mathrm{F}$ and $\mathrm{S}$, there are five potentially different phenotypes for a monomeric enzyme as given in Fig. 1 . The numbers $(1-4)$ next to the bands indicate the relative proportions of enzyme molecules that constitute the different bands. However, because straining in- tensity may vary from plant to plant and from allele to allele, even when the proportions of molecules are constant, distinguishing the three two-banded phenotypes from staining intensities is often unreliable. First, the use of selfed progeny arrays to distinguish between the various two-banded phenotypes in a population survey is outlined. Then the determination of estimates of selffertilization and allelic frequencies in population samples, which have only three categories, FF FF, SS SS, and the remaining category, FS, composed of all those genotypes with two-banded phenotypes is shown. (In this symbolism, the first two letters refer to

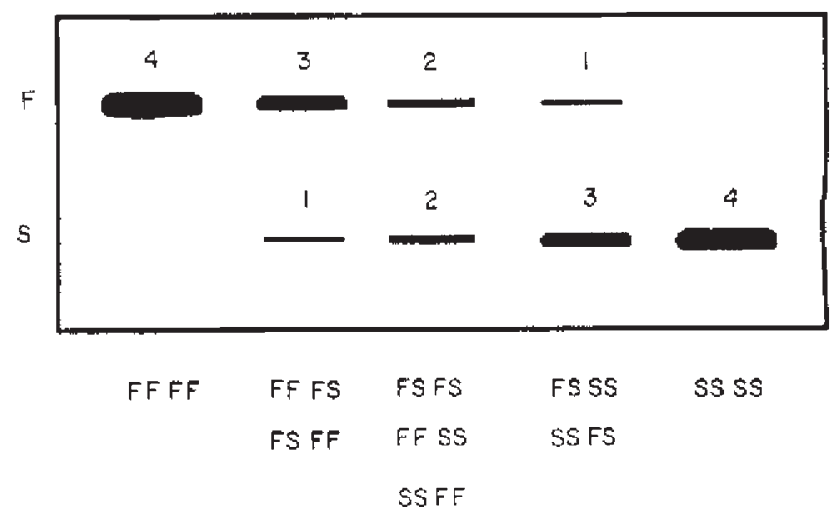

Fig. 1 Variability at two isoloci shown as the five possible electrophoretic phenotypes observed for a monomeric enzyme segregating for fast $(\mathrm{F})$ and slow (S) alleles in both genomes. The genotypes that can result in the various phenotypes are shown below. The numbers adjacent to the bands refer to the relative number of molecules constituting them. 
the genotype in the first genome, the last two to that in the second genome, and $\mathrm{F}$ and $\mathrm{S}$ indicate fast and slow alleles, respectively.)

\section{Population survey}

As in the Menzies' wallflower data discussed later, the population may be surveyed for only three categories, the homozygous genotypes FF FF and SS SS, and the remaining category which is composed of the remaining genotypes. It is advisable, if possible, to examine selfed progeny arrays to separate out more categories as shown below, but this may not always be feasible.

First, in examining selfed progeny arrays, ignore coupling and repulsion differences in the double heterozygote, FS FS, because there is assumed to be independent assortment between the two genomes. As a result, the nine possible maternal genotypes can be grouped into six classes based on the type of progeny arrays produced upon self-fertilization (Table 1). For example, genotypes FF FS and FS FF are expected to have one-quarter FF FF progeny and three-quarters progeny with both $F$ and $S$ alleles, denoted as the general FS category here.

The following procedure can be used to determine the different maternal genotypes. From a given genotypic class, let the expected proportions of progeny types $\mathrm{FF} \mathrm{FF}, \mathrm{FS}$, and SS SS be $P_{\mathrm{FF}}, P_{\mathrm{FS}}$, and $P_{\mathrm{SS}}$, respectively. If the observed numbers of the three types are $N_{\mathrm{FF}}, N_{\mathrm{FS}}$, and $N_{\mathrm{SS}}$, then the likelihood of such an array from genotype class $i$ is proportional to

$L(i)=P_{\mathrm{FF}}^{N_{\mathrm{FF}}} P_{\mathrm{FS}}^{N_{\mathrm{Fs}}} P_{\mathrm{SS}}^{N_{\mathrm{SS}}}$.

However, if $N$ progeny are sampled from a given maternal plant and progeny types are observed that

Table 1 The proportions of different types of progeny produced by self-fertilization of the given maternal genotypes

\begin{tabular}{llcc}
\hline \multirow{4}{*}{$\begin{array}{l}\text { Maternal } \\
\text { genotype }\end{array}$} & \multicolumn{2}{l}{ Progeny genotype or phenotype } \\
\cline { 2 - 4 } FF FF & 1 & FS & SS SS \\
\hline (1) FF FF & 1 & - & - \\
(2) FF FS & $\frac{1}{4}$ & $\frac{3}{4}$ & - \\
FS FF & & 1 & - \\
(3) FF SS & - & & \\
SS FF & & $\frac{7}{8}$ & $\frac{1}{16}$ \\
(4) FS FS & $\frac{1}{16}$ & $\frac{3}{4}$ & $\frac{1}{4}$ \\
(5) FS SS & - & - & 1 \\
SS FS & & - & \\
(6) SS SS & - & & \\
\hline
\end{tabular}

exclude certain maternal genotypes, this likelihood should not have to be calculated for the excluded types. For example, if both FF FF and FS progeny are observed, then the matemal parent is either of class 2 or 4 (the other four maternal classes may be excluded). The likelihood ratios, $L(2) / L(4)$ and $L(4) / L(2)$ can then be calculated and if the $\log _{10}$ of either is greater then 3 , then the class in the numerator of that ratio can be considered the maternal parent (following Conneally $e t$ al., 1985). The population frequencies for the six classes can be determined by examining progeny arrays for a random sample of maternal plants from a population and determining the phenotypic class for each maternal plant in this manner.

\section{Expected genotypic frequencies}

When there is partial self-fertilization in a population, then the frequency of genotypes at equilibrium in a population determined by two unlinked loci is given by Bennett \& Binet (1956). The deviation from the frequency expected from the product of the equilibrium frequencies at the separate loci is a function of

$d=\frac{4 s(1-s) p_{1} q_{1} p_{2} q_{2}}{(4-s)(2-s)^{2}}$

where $s$ is the proportion of self-fertilization and $p_{1}$ and $q_{1}$ are the frequencies of the $\mathrm{F}$ and $\mathrm{S}$ alleles in genome 1 and $p_{2}$ and $q_{2}$ are the frequencies of the $\mathrm{F}$ and $S$ alleles in the second genome. The expected frequencies of the six classes of genotypes in a population given in Table 1 are then

$$
\begin{aligned}
& G_{1}=\left(p_{1}^{2}+\frac{s p_{1} q_{1}}{2-s}\right)\left(p_{2}^{2}+\frac{s p_{2} q_{2}}{2-s}\right)+d \\
& G_{2}=\left(p_{1}^{2}+\frac{s p_{1} q_{1}}{2-s}\right)\left(\frac{4 p_{2} q_{2}(1-s)}{2-s}\right) \\
& +\left(\frac{4 p_{1} q_{1}(1-s)}{2-s}\right)\left(p_{2}^{2}+\frac{s p_{2} q_{2}}{2-s}\right)-4 d \\
& G_{3}=\left(p_{1}^{2}+\frac{s p_{1} q_{1}}{2-s}\right)\left(q_{2}^{2}+\frac{s p_{2} q_{2}}{2-s}\right) \\
& +\left(q_{1}^{2}+\frac{s p_{1} q_{1}}{2-s}\right)\left(p_{2}^{2}+\frac{s p_{2} q_{2}}{2-s}\right)+2 d \\
& G_{4}=\left(\frac{4 p_{1} q_{1}(1-s)}{2-s}\right)\left(\frac{4 p_{2} q_{2}(1-s)}{2-s}\right)+4 d
\end{aligned}
$$




$$
\begin{aligned}
G_{5}= & \left(\frac{4 p_{1} q_{1}(1-s)}{2-s}\right)\left(q_{2}^{2}+\frac{s p_{2} q_{2}}{2-s}\right) \\
& +\left(q_{1}^{2}+\frac{s p_{1} q_{1}}{2-s}\right)\left(\frac{4 p_{2} q_{2}(1-s)}{2-s}\right)-4 d \\
G_{6}= & \left(q_{1}^{2}+\frac{s p_{1} q_{1}}{2-s}\right)\left(q_{2}^{2}+\frac{s p_{2} q_{2}}{2-s}\right)+d
\end{aligned}
$$

all of which are a function of $s, p_{1}$ and $p_{2}\left(q_{1}=1-p_{1}\right.$ and $q_{2}=1-p_{2}$ ). If a population survey, using the approach outlined in the previous section for the six genotypic categories, obtains $N_{i}$ individuals for the $i$ th genotypic class, then the likelihood of observing this array in a population is

$L_{G}=\Pi G_{i}^{N_{j}}$.

Notice in this case there are five independent genotypic categories and three parameters to estimate. If only three different classes (FF FF, SS SS, and FS) are distinguishable because the FS class has not been subdivided by examing selfed progeny arrays, then

$L_{G}=G_{1}^{N_{1}} G_{6}^{N_{6}}\left(1-G_{1}-G_{6}\right)^{N-N_{1}-N_{6}}$

where $N$ is the total number of individuals in the genotypic survey and $N_{1}$ and $N_{6}$ are the numbers of FF FF and SS SS genotypes observed. In this case, because there are only two independent categories and three parameters, no estimation is possible.

\section{Direct estimation of self-fertilization}

Assuming segregation and independent assortment, we can obtain the frequencies of pollen gametes produced

Table 2 The gametic frequencies in the pollen produced by the given paternal genotype

\begin{tabular}{rllll}
\hline & & \multicolumn{2}{l}{ Pollen } & \\
\cline { 3 - 5 } $\begin{array}{l}\text { Paternal } \\
\text { genotype }\end{array}$ & Frequency & FF & FS or SF & SS \\
\hline (1) FF FF & $G_{1}$ & $G_{1}$ & - & - \\
(2) FF FS & $G_{2}$ & $\frac{1}{2} G_{2}$ & $\frac{1}{2} G_{2}$ & - \\
FS FF & & & & \\
(3) FF SS & $G_{3}$ & - & $G_{3}$ & - \\
SS FF & & & & \\
(4) FS FS & $G_{4}$ & $\frac{1}{4} G_{4}$ & $\frac{1}{2} G_{4}$ & $\frac{1}{4} G_{4}$ \\
(5) FS SS & $G_{5}$ & - & $\frac{1}{2} G_{5}$ & $\frac{1}{2} G_{5}$ \\
SS FS & & & & \\
(6) SS SS & $G_{6}$ & - & - & $G_{6}$ \\
\hline
\end{tabular}

by the six different genotypic classes (see Table 2). Therefore, the frequency of the three types of pollen is

$$
\begin{aligned}
& \begin{aligned}
f(\mathrm{FF}) & =G_{1}+\frac{1}{2} G_{2}+\frac{1}{4} G_{4} \\
& =p_{1} p_{2}
\end{aligned} \\
& \begin{aligned}
f(\mathrm{FS} \text { or } \mathrm{SF}) & =G_{3}+\frac{1}{2}\left(G_{2}+G_{4}+G_{5}\right) \\
& =1-p_{1} q_{2}-q_{1} p_{2}
\end{aligned} \\
& \begin{aligned}
f(\mathrm{SS})= & G_{6}+\frac{1}{2} G_{5}+\frac{1}{4} G_{4} \\
= & q_{1} q_{2} .
\end{aligned}
\end{aligned}
$$

If we examine only progenies from homozygous FF FF and SS SS maternal plants, then the expected frequencies of progeny can be obtained from Table 3 . [Note that this procedure could be expanded to include other maternal genotypes as shown by Ritland \& Jain (1981) or Hedrick \& Ritland (1990) although the FF FF and SS SS maternal plants are generally the most informative.] Therefore, the expected proportion of progeny different from the maternal FF FF genotype is

$$
\begin{aligned}
H_{\mathrm{F}} & =(1-s)[f(\mathrm{FS})+f(\mathrm{SF})+f(\mathrm{SS})] \\
& =(1-s)\left(1-p_{1} p_{2}\right) .
\end{aligned}
$$

If we observe $N_{\mathrm{FF}}$ homozygous FF FF progeny and $N_{\mathrm{FS}}$ outcrossed progeny, then the likelihood of such an array is

$L_{\mathrm{F}}=\left(1-H_{\mathrm{F}}\right)^{N_{\mathrm{FF}}}\left(H_{\mathrm{F}}\right)^{N_{\mathrm{FS}}}$.

Likewise for the SS SS maternal genotype, the expected proportion of progeny that are not SS SS is

$H_{\mathrm{S}}=(1-s)\left\langle 1-q_{1} q_{2}\right)$.

The likelihood of such an array is

$L_{\mathrm{S}}=\left(1-H_{\mathrm{S}}\right)^{N_{\mathrm{SS}}}\left(H_{\mathrm{S}}\right)^{N_{\mathrm{rS}}}$

Table 3 The expected progeny proportions from FF FF and SS SS maternal genotypes

Maternal Mating genotype proportions Progeny

(a)

FF FF FFFS FSFF FS FS

FF FF $s$

$1-s$

$1-\quad-$

$f(\mathrm{FF}) \quad f(\mathrm{FS}) \quad f(\mathrm{SF})$

$f(\mathbf{S S})$

(b)

SS SS FSSS SSFS FS FS

ss ss

\begin{tabular}{llll}
\hline 1 & - & - & - \\
$f(\mathrm{SS})$ & $f(\mathrm{FS})$ & $f(\mathrm{SF})$ & $f(\mathrm{SS})$
\end{tabular}


where $N_{\mathrm{SS}}$ and $N_{\mathrm{FS}}$ are the number of homozygous SS $\mathrm{SS}$ and outcrossed progeny.

\section{Joint estimation}

If it is assumed that the progeny arrays from FF FF and SS SS parents and the population data from the genotypic survey are independent, then the joint likelihood of the arrays seen in expressions $4,6 \mathrm{~b}$, and $7 \mathrm{~b}$ is

$L=L_{G} L_{\mathrm{F}} L_{\mathrm{S}}$.

Notice that $L_{G}, L_{\mathrm{F}}$, and $L_{\mathrm{S}}$ are all functions of the same three parameters: $p_{1}, p_{2}$, and $s$. If we assume the arrays used for these different likelihoods are independent of each other, then expression (8) has four independent categories to estimate these three parameters. If data are available on progeny arrays from only one type of homozygous maternal parent, then estimation would also be possible because there would be three independent categories to estimate three parameters.

\section{An example}

Erysimum menziesii is a rare polyploid mustard found in a few isolated sites in the dunes along the California coast. Phenotypes on starch gels show that a pair of duplicated loci for shikimate dehydrogenase ( $\mathrm{ShDH}$ ) govern bands resolved in the slower region of the gel. In most of the populations of E. menziesii in Humboldt County, phenotypic data suggest that $S$ is fixed in one genome and $S$ and $F$ segregate in the other, with $F$ at much higher frequencies. The distributions of phenotypes make it probable that the common phenotype FS is mostly represented by the doubly homozygous FF SS genotype.

In the southernmost Humboldt County population, however, both possible single-banded types (FF FF and SS SS) have been observed, giving evidence that both alleles are present in both genomes. A genotypic survey of 74 individuals in this population was conducted in 1989 and progeny arrays were examined from 13 FF FF and 4 SS SS maternal plants found in this survey (these data are summarized under Observed in Table 4). With this information, we can calculate the likelihood under various combinations of allelic frequencies and self-fertilization rates (all combinations of $s, p_{1}$, and $p_{2}$ were calculated at 0.01 intervals to find the maximum). With input from all three datasets, the $\log _{10}$ probability value (using the appropriate binomial and trinomial coefficients) for expression (8) is maximum (and equal to -7.31 ) when $s=0.50$ and $p_{1}=p_{2}=0.59$. Using these values of $s, p_{1}$, and $p_{2}$, the expected numbers in the various categories were calculated using expressions $3,6 \mathrm{a}$, and $7 \mathrm{a}$ (see Expected column of Table 4).

Ordinarily, the 95 per cent confidence interval is within one lod score value of the maximum where the lod score is the ratio of maximum log likelihood score divided by that for a null hypothesis (e.g. Conneally $e t$ al., 1985). [A less conservative alternative is two $\log _{e}$ likelihood units (Edwards, 1972).] The obvious null hypothesis is that $s=0$ (random mating). When all the datasets are combined, then the maximum log probability for $s=0$ is -57.90 (here $p_{1}=p_{2}=0.42$ ), many orders of magnitude less than the -7.31 value when $s=0.50$ and $p_{1}=p_{2}=0.59$. In other words, the null hypothesis of no self-fertilization is easily rejected.

Another way to visualize the extent of dependence of the likelihood values on the parameters is to find the combinations of parameters that give likelihood scores a given magnitude below the maximum. Figure 2 indicates the parameter combination with the maximum likelihood score (closed circle) and the ellipse of parameter values which give a likelihood value $10^{-3}$ that of the maximum. Obviously, even with these confidence intervals and under the assumption that the allelic frequencies are equal, the confidence region around the selfing rate estimate is quite small. The region of parameter values $10^{-3}$ that of the maximum, given that $s=0.5$, for various combinations of allelic frequencies at the two loci, is much larget (Fig. 3). This may be related to a similar finding by Waples (1988) in which

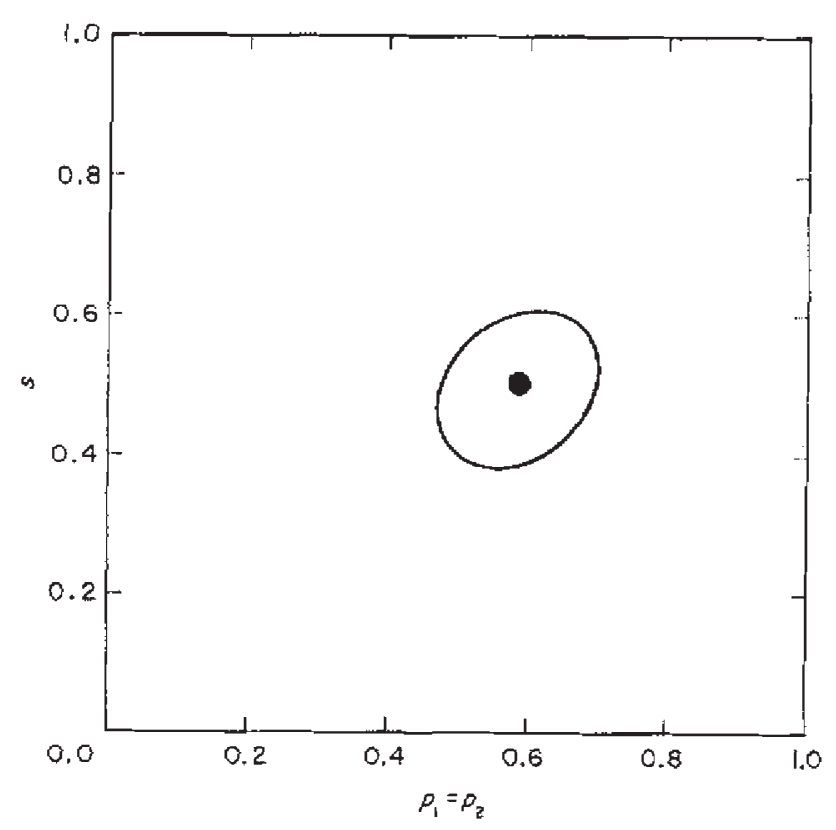

Fig. 2 The combinations of $s$ and $p_{1}=p_{2}$ (shown by the ellipse) that give likelihood values $10^{-3}$ times the maximum likelihood score (closed circle). 


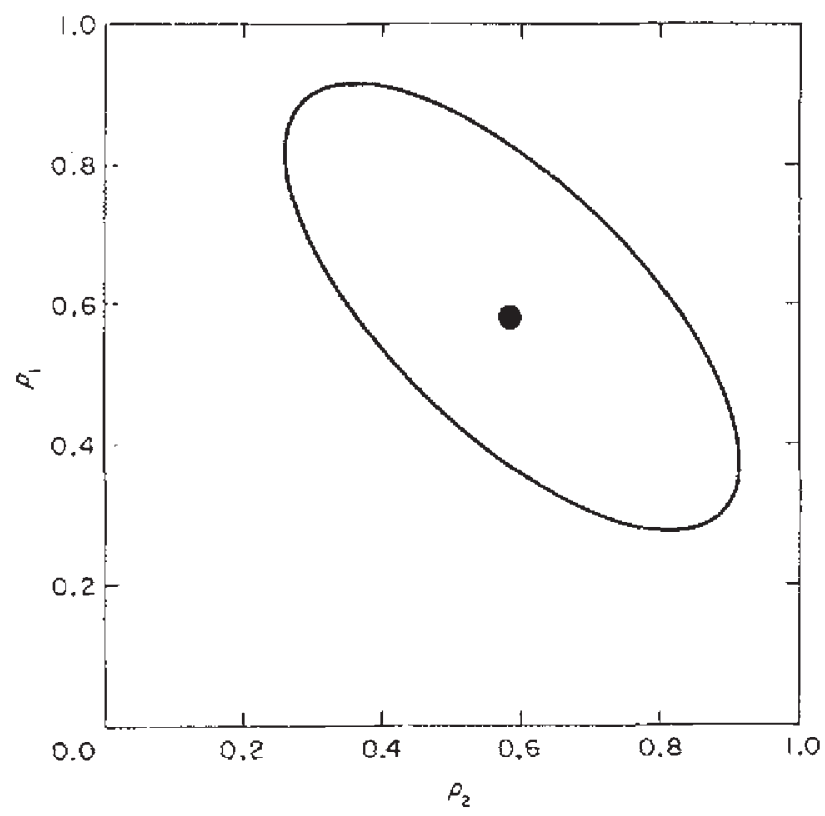

Fig, 3 The combinations of $p_{1}$ and $p_{2}$ (shown by the ellipse) given that $s=0.5$ that give a likelihood value $10^{-3}$ times the maximum likelihood score (closed circle).

he suggests that allelic frequency estimates in tetraploids may have broad confidence limits.

A goodness of fit calculation for the observed and expected numbers in Table 4 indicates highly significant deviations $\left(\chi^{2}=20.9\right.$, d.f. $\left.=4, P<0.01\right)$. The

Table 4 The observed and expected genotypic numbers of a genotypic survey and progeny numbers from FF FF and SS SS maternal genotypes from $E$. menziesii. The progeny arrays for FF FF and SS SS are the summation of 13 and 4 maternal plants, respectively. The expected numbers were calculated using the maximum likelihood estimates of $s, p_{1}$, and $p_{2}$

\begin{tabular}{lrr}
\hline & Observed & Expected \\
\hline Genotypic survey & & \\
$N_{1}$ & 23 & 14.2 \\
$N-N_{1}-N_{6}$ & 46 & 54.7 \\
$N_{6}$ & 5 & 5.1 \\
Progeny arrays & & \\
FF FF maternal genotype & & \\
$\quad N_{\mathrm{FF}}$ & 192 & 212.3 \\
$F_{\mathrm{FS}}$ & 123 & 102.7 \\
SS SS maternal genotype & & \\
$N_{\mathrm{SS}}$ & 50 & 64.8 \\
$\quad N_{\mathrm{FS}}$ & 61 & 46.2 \\
\hline
\end{tabular}

observed number is greater than expected for the homozygote $\mathrm{FF} \mathrm{FF}$ in the genotypic survey and the observed numbers in the homozygote classes are lower than expected in the progeny arrays. These differences are reminiscent of findings in some diploids with high outcrossing rates in which the estimated rate of selfing is higher using genotypic surveys than for progeny arrays, part of the 'heterozygosity paradox' (e.g. Brown, 1979; Hedrick \& Cockerham, 1986). Despite this, the estimates obtained here appear to be a compromise between those that would best fit the genotypic survey or the progeny arrays.

Perhaps these deviations may be part of the reason why the maximum likelihood estimate gave equal allelic frequencies at the two loci. Waples (1988) found that when there is no solution that is a particularly good fit for the data that the solution with equal allelic frequencies will commonly be the maximum likelihood solution.

We planned to continue examination of the ShDH polymorphism in this population but a survey in 1990 gave a low frequency of anomalous electrophoretic phenotypes in which neither the $F$ or $S$ band was observed. Two possible explanations for this finding are that there is a null allele in both genomes or a third allele in both genomes that is masked by an invariant band anodal to the Fast-Slow region. Although we did not see any of this phenotype in the 1989 cohort, a third allele of this sort may upwardly bias our selfing estimate. In other words, although these and other data suggest a significant selfing proportion in this Menzies' wallflower population, we feel that the actual estimate calculated above must be taken tentatively.

\section{Discussion}

Isoloci are duplicated loci that share alleles whose gene products have identical electrophoretic mobilities (e.g. Waples, 1988). Isoloci typify autopolyploids and allopolyploids derived from ancestral species with substantial genetic similarity. In autopolyploids, diploidization (typical Mendelian disomic inheritance) may subsequently develop. In allopolyploids, disomic inheritance is expected even when the ancestral species are closely related. Furthermore, instances in which gene duplication produces a second locus are examples of isoloci. If subsequently these duplicated loci are separated by some chromosomal event, then they would also be isoloci showing disomic inheritance.

Many plants and a number of invertebrates have substantial rates of self fertilization. If independently assorting isoloci are present in these organisms, then the techniques developed here are appropriate for 
estimating allelic frequencies and the rate of self-fertilization in them. Obviously these organisms may not be ideal ones in which to study mating systems or other evolutionary phenomena but, as in our study of Menzies' wallflower, interest in an organism may be dictated by other considerations, such as its endangered species status.

Note that we have assumed that the population being considered is at genotypic equilibrium and that only the mating system influences genotypic frequencies. The problems with these assumptions in diploids have been widely discussed (see Brown, 1979; Brown et al., 1985; Hedrick, 1990 for reviews) and most of these problems we assume to be similar in diploidized polyploids. One additional problem occurs in that three inbreeding coefficients, not just one, are necessary to describe the genotypic frequencies if the population is not at equilibrium (e.g. Weir \& Cockerham, 1973).

\section{Acknowledgements}

Phil Hedrick was a Distinguished Research Fellow at Bodega Marine Laboratory while developing this work. We appreciate the comments of Kermit Ritland and Robin Waples on earlier versions of the manuscript. The Louisiana Pacific Corporation and the Simpson Paper Company supported part of this work through the Menzies' Wallflower Habitat Preservation Program, which was mandated by the Environmental Protection Agency as part of a mitigation decision.

\section{References}

BENNETT, J. H. AND BINET, F. E. 1956. Association between Mendelian factors with mixed selfing and random mating. Heredity, 10, 51-56.

BROWN, A. H. D. 1979. Enzyme polymorphism in plant populations. Theoret. Pop. Biol, 15, 1-42.

BROWN, A. H. D., BARRETT, S. C. H. AND MORAN, G. F. 1985. Mating system estimation in forest trees: models, methods, and meanings. In: Gregorius, H.-K. (ed.) Population Genetics in Forestry. Lecture Notes in Biomathematics 60 . SpringerVerlag, Berlin, pp. 32-49.

CONNEALLY, J. H., EDWARDS, J. H., KIDD, K. K., LALOUEL, J. M., MORTON, N. E., OTT, J. AND WHITE, R, 1985. Report of the committee on methods of linkage analysis and reporting. Cytogenet. Cell Biol., 40, 356-359.

RDWARDS, A. W. F. 1972. Likelihood. Cambridge University Press, Cambridge.

HEDRICK, P. W. 1990. Mating systems and evolutionary genetics. In: Wohrmann, K. and Jain, S. (eds) Population Biology: Ecological and Evolutionary Viewpoints. Springer-Verlag, Berlin, pp. 83-114.

HEDRICK, P. W. AND COCKERHAM, C. C. 1986. Partial inbreeding: equilibrium heterozygosity and the heterozygosity paradox. Evolution, 40, 856-861.

HEDRICK, P. W. AND RITLAND, K. 1990. Gametic disequilibrium and multilocus estimation of selfing rates. Heredity, 65 , 343-347.

RITLAND, K. AND JAIN, s. 1981. A model for the estimation of outcrossing rate and gene frequencies using independent loci. Heredity, 47, 35-52.

WAPLES, R. S. 1988. Estimation of allele frequencies at isoloci. Genetics, 118, 371-384.

WEIR, B. S. AND COCKERHAM, c. c. 1973. Mixed self and random mating at two loci. Genet. Res., 21, 247-262. 